\title{
Impacto da COVID-19 na Segurança Alimentar e Nutricional: desafios e estratégias
}

\section{emergentes}

\author{
Impact of COVID-19 on Food and Nutritional Security: emerging challenges and Strategies \\ Impacto del COVID-19 en la Seguridad Alimentaria y Nutricional: desafíos y estratégias
}

emergentes

Recebido: 21/09/2021 | Revisado: 02/10/2021 | Aceito: 03/10/2021 | Publicado: 04/10/2021

\author{
Ana Gabriella Costa Lemos da Silva \\ ORCID: https://orcid.org/0000-0001-7761-2708 \\ Universidade Federal do Rio Grande do Norte, Brasil \\ E-mail: gabriella_lemos_06@yahoo.com.br \\ Tatiana Maria de Oliveira \\ ORCID: https://orcid.org/0000-0001-6401-1938 \\ Universidade Federal do Rio Grande do Norte, Brasil \\ E-mail: tatianam_oliveira@hotmail.com \\ Aylen Evelin Mendoza Cáceres \\ ORCID: https://orcid.org/0000-0002-6286-0677 \\ Universidade Federal do Rio Grande do Norte, Brasil \\ E-mail: aylen.mc93@gmail.com \\ Karla Danielly da Silva Ribeiro \\ ORCID: https://orcid.org/0000-0002-2251-5967 \\ Universidade Federal do Rio Grande do Norte, Brasil \\ E-mail: karladaniellysr@yahoo.com.br \\ Severina Carla Vieira Cunha Lima \\ ORCID: https://orcid.org/0000-0001-8268-1986 \\ Universidade Federal do Rio Grande do Norte, Brasil \\ E-mail: scvclima@gmail.com \\ Clélia de Oliveira Lyra \\ ORCID: https://orcid.org/0000-0002-1474-3812 \\ Universidade Federal do Rio Grande do Norte, Brasil \\ E-mail: clelia_lyra@yahoo.com.br
}

\begin{abstract}
Resumo
Objetivo: discutir sobre o impacto da pandemia da COVID-19 na segurança alimentar e nutricional (SAN) das populações, mostrando os desafios enfrentados e quais as estratégias emergentes dos países para impedir a expansão da insegurança alimentar durante e após essa crise social e sanitária. Metodologia: revisão narrativa realizada através de uma busca bibliográfica de artigos em português e inglês, disponíveis nas bases de dados PubMed, Scielo, Embase e Lilacs. Foram incluídos artigos publicados sem data limite de publicação e os quais se encontravam disponíveis na íntegra. Os descritores utilizados foram: "Segurança alimentar e nutricional", "Insegurança alimentar", "Pandemia" e "COVID-19". Resultados: medidas para controlar os surtos da COVID-19 afetaram as cadeias de abastecimento de alimentos. Além disso, altos níveis de desemprego, perda de renda e aumento dos custos dos alimentos também dificultaram o acesso aos alimentos, principalmente, para a população mais vulnerável socialmente. Os processos de disponibilidade e acesso aos alimentos durante a pandemia têm impactado diretamente na escolha, preparo e consumo alimentar da população. Desta forma, medidas governamentais devem ajudar a promover a SAN das populações, por meio da criação ou expansão dos programas de proteção social, distribuição e melhoria no acesso aos alimentos, proteção de empregos e meios de subsistência e apoio aos pequenos produtores locais. Conclusão: As ações promovidas pelos governos devem garantir a SAN das populações, assegurando o acesso à alimentação adequada e saudável e objetivando a redução de impactos negativos promovidos pelo SARS-CoV-2 na condição da alimentação, saúde e nutrição da população, principalmente dos mais vulneráveis.
\end{abstract}

Palavras-chave: Segurança alimentar e nutricional; COVID-19; Pandemia por COVID-19.

\begin{abstract}
Objectives: To discuss the impact of the COVID-19 pandemic on the food and nutrition security (FNS) of populations, showing the challenges faced and the emerging tactics of countries to prevent the expansion of food insecurity during and after this social and health crisis. Methodology: Narrative review carried out through a bibliographic search of articles in Portuguese and English, available in PubMed, Scielo, Embase and Lilacs databases. Articles published without publication deadline and which were available in full were included. The descriptors used were: "Food and nutrition security", "Food insecurity", "Pandemic" and "COVID-19". Results: Procedures to control
\end{abstract}


COVID-19 outbreaks affected food supply chains. In addition, high levels of unemployment, loss of income and rising food costs also hampered access to food, especially for the most socially vulnerable population. The processes of availability and access to food during the pandemic have had a direct impact on the population's food choice, preparation and consumption. Consequently, government measures should help to promote FNS among populations, through the creation or expansion of social protection programs, distribution and improvement in access to food, protection of jobs and livelihoods, and support for small local producers. Conclusion: The actions promoted by governments must guarantee the FNS of populations, ensuring access to adequate and healthy food and aiming to reduce the negative impacts promoted by SARS-CoV-2 on the condition of food, health and nutrition of the population, especially the most vulnerable.

Keywords: Food and nutrition security; COVID-19; COVID-19 Pandemic.

\section{Resumen}

Objetivo: discutir el impacto de la pandemia COVID-19 en la Seguridad Alimentaria y Nutricional (SAN) de las poblaciones, dando a conocer los desafíos enfrentados y las estrategias emergentes de los países para prevenir la expansión de la inseguridad alimentaria durante y después de esta crisis social y sanitaria. Metodología: revisión narrativa realizada a través de una búsqueda bibliográfica de artículos en portugués e inglés, disponibles en la base de datos PubMed, Scielo, Embase e Lilacs. Fueron incluidos artículos publicados sin fecha límite de publicación y que además estaban disponibles en su totalidad. Los descriptores utilizados fueron: "Seguridad alimentaria y nutricional", "Inseguridad alimentaria", "Pandemia" y "COVID-19". Resultados: Las medidas para controlar los brotes de COVID19 afectaron las cadenas de suministros de alimentos. Además, los altos niveles de desempleo, la perdida de ingresos y el aumento de los costos de alimentos también obstaculizaron el acceso a los alimentos, especialmente, para la población más vulnerable socialmente. Los procesos de disponibilidad y acceso a los alimentos durante la pandemia han tenido un impacto directo en la elección, preparación y consumo de alimentos de la población. De tal manera, las medidas gubernamentales deben ayudar a promover la SAN entre las poblaciones, a través de la creación o expansión de programas de protección social, la distribución y mejora del acceso a los alimentos, la protección del empleo y los medios de subsistencia, y el apoyo a los pequeños productores locales. Conclusión: Las acciones impulsadas por los gobiernos deben garantizar la SAN de las poblaciones, asegurando el acceso a una alimentación adecuada y saludable, con el objetivo de reducir los impactos negativos promovidos por el SARS-CoV-2 sobre la condición de alimentación, salud y nutrición de la población, especialmente la más vulnerable.

Palabras clave: Seguridad alimentaria y nutricional; COVID-19; Pandemia por COVID-19.

\section{Introdução}

A pandemia por COVID-19 ainda trará diversos impactos socioambientais, políticos e econômicos de forma distinta entre os povos. Desde que decretada pela Organização Mundial de Saúde (OMS), a comunidade científica trabalha coletivamente para definir e encontrar estratégias de enfrentamento que minimizem os efeitos e a rápida propagação da doença (WHO, 2021a). A COVID-19 é causada pelo vírus SARS-CoV-2 e transmitida principalmente pelo ar ou por contato direto com partículas de saliva ou secreções respiratórias, podendo também contaminar mãos e superfícies. Devido a essa característica, o distanciamento social foi considerado pela OMS a medida mais eficaz para conter a propagação da doença, sendo uma estratégia utilizada mundialmente (WHO, 2021a).

É importante destacar que o distanciamento social e/ou físico provocou a suspensão/distanciamento de diversas atividades laborais, como postos de trabalho, escolas, universidades, alterando a rotina de populações para essa nova forma de convivência. Considerando a importância do isolamento social associado ao fato da maioria das pessoas estarem em casa, essa pandemia tem revelado a importância de ações das autoridades locais para o sucesso de seu enfrentamento, que vão desde poder econômico a eficiência política, estruturação da saúde (recursos humanos, rede e insumos) e menor desigualdade social. Salienta-se que países economicamente e politicamente mais organizados, com melhores indicadores de saúde e redes de saúde eficientes conseguiram achatar a curva de contágio e reduzir os impactos decorrentes dessa pandemia com maior eficácia (WHO, 2021b).

O continente americano é o mais afetado pela pandemia, principalmente os Estados Unidos e Brasil (WHO, 2021b). Esses países apresentam como características comuns o perfil desigual de expansão da doença, que acomete principalmente grupos vulneráveis economicamente e socialmente, elevando o risco da pobreza e impactos sociais.

Diante disso, este estudo objetiva refletir sobre o impacto dessa crise além da saúde individual: como ela pode 
impactar na segurança alimentar e nutricional (SAN) e quais estratégias emergentes vêm sendo realizadas para evitar a nova crise pós-pandemia - a da fome e a miséria.

\section{Metodologia}

Esse artigo é resultado de uma revisão da literatura narrativa sobre o impacto da pandemia da COVID-19 na segurança alimentar da população, a partir do levantamento de informações, estudos, leituras e análises de opiniões apontadas por diferentes autores, bem como a interpretação desses estudos, destacando a relevância do assunto em questão. Os artigos de revisão narrativa são publicações amplas, apropriadas na descrição e discussão do desenvolvimento ou do estado da arte de um determinado assunto, sob ponto de vista teórico e até mesmo contextual. Após a busca e análise na literatura, o autor deve interpretar e fazer a análise crítica pessoal (Rother, 2007).

Desta forma, o presente trabalho fundamentou-se em uma pesquisa de revisão da literatura narrativa realizada através de uma busca bibliográfica de artigos em português e inglês, disponíveis nas bases de PubMed, Scielo, Embase e Lilacs. Foram incluídos artigos disponíveis na íntegra e sem data limite de publicação. Os descritores utilizados para construir o estudo e pesquisa foram: "Segurança alimentar e nutricional”, "Insegurança alimentar", "Pandemia” e "COVID-19”. Além disso, foram incluídos relatórios e documentos oficiais que apresentavam relevância ao tema. Após análise seletiva e criteriosa dos dados coletados, foram excluídos da revisão artigos infundamentados e/ou que não obedeciam às especificações buscadas. Após a identificação dos textos, realizou-se uma leitura e análise criteriosas a fim de relacionar os textos que compuseram a revisão da literatura ao tema proposto.

\section{Resultados e Discussão}

\section{Desigualdades sociais e iniquidades em saúde}

$\mathrm{O}$ arranjo da incidência do coronavírus, entre os territórios e indivíduos, aparece como prioridade a ser considerada. O Sars-CoV-2 foi trazido do exterior para o Brasil por estratos mais privilegiados ou médios que estão localizados particularmente em regiões mais desenvolvidas (Santos, 2020). Ressalta-se que essa população teve maior acesso aos métodos de diagnóstico da doença, não ocorrendo a testagem em massa no nosso país. Outrossim, a condição de caráter socioeconômico influencia o grau de exposição aos fatores de risco e evolução do coronavírus: pessoas de estrato socioeconômico inferior tendem a estar em desvantagem no amplo conjunto de fatores que medeiam a relação entre as condições sociais e a doença (House \& Williams, 2000).

Diante disso, a pandemia por COVID-19 intensificou as desigualdades sociais, econômicas, políticas e culturais existentes no mundo. A doença atinge majoritariamente os grupos sociais mais vulneráveis, como idosos, pobres, moradores de rua e habitantes de áreas com precárias infraestruturas urbanas e de saúde, sem acesso às condições básicas para o enfrentamento da doença (Quinzani, 2020).

Frente a esse quadro, é perceptível que a sobrevivência para milhões de pessoas no Brasil vem do subemprego e com menos de 145 reais por mês. Boas condições de higiene, saúde, isolamento social e boa alimentação tornam-se árduas para essa parte da população. Consonante, Bartley (2017) afirma que o modo de compreender a desigualdade social tem implicações relevantes para o estudo da desigualdade em saúde, uma vez que as desigualdades são de diferentes tipos e podem influenciar a saúde de formas distintas (Bartley, 2017).

A CEPAL e a Organização Internacional do Trabalho indicam que a pandemia tem gerado efeitos negativos sobre o mercado de trabalho, com consequências nos setores formais (redução de horas e de salários e demissões) e informais (queda de emprego) devido ao distanciamento social e às restrições de circulação (CEPAL, 2020a). Diante disso, essas pessoas precisam ser assistidas com políticas públicas de emprego e transferência de renda, políticas que as protejam da fome e da 
pobreza, e não se limitem aos auxílios emergenciais. No longo prazo, os países precisam de uma política de desenvolvimento que implique no aumento dos gastos em programas sociais já existentes, no sentido de proteger milhões de trabalhadores que vivem na informalidade e residem em comunidades ou assentamentos precários, sendo importante lembrar também da população de rua.

Outro enfoque do enfrentamento da pandemia, refere-se à capacidade de atendimento nos serviços de saúde. Em 2020, no Brasil, as hospitalizações pela síndrome respiratória aguda grave superaram o observado no mesmo período dos 10 anos anteriores (Bastos, 2020). O aumento das hospitalizações, a falta de informação específica sobre o agente etiológico das hospitalizações e a predominância de casos entre idosos e pessoas com comorbidades sobrecarregaram o sistema de saúde.

Ademais, como consequência da forte recessão econômica na América Latina, que registrou uma queda do PIB de 7,7\%, estima-se que em 2020 a taxa da extrema pobreza se situou em 12,5\% e a taxa da pobreza atingiu 33,7\% da população. Isso significa que o total de pessoas pobres chegou a 209 milhões no final de 2020, 22 milhões de pessoas a mais do que no ano anterior. Desse total, 78 milhões de pessoas estavam em situação de extrema pobreza, 8 milhões a mais do que em 2019. Além disso, a pobreza é maior nas áreas rurais, entre crianças e adolescentes; indígenas e afrodescendentes; e na população com menores níveis educativos (CEPAL, 2020b). Portanto, a dinâmica da pobreza e da desigualdade mundial está sendo afetada negativamente. Somado ao aumento do desemprego e queda da renda, milhões de pessoas não estão conseguindo adquirir alimentos suficientes e muitas outras optam por alimentos mais baratos e de menor qualidade nutricional.

\section{Disponibilidade e acesso aos alimentos}

Além dos efeitos epidemiológicos, sociais e econômicos, a crise global decorrente da pandemia por COVID-19 tem exposto a fragilidade dos sistemas alimentares. Os esforços mundiais para controlar o vírus, limitando o movimento de pessoas, estão inevitavelmente causando choques econômicos e custos sociais que afetam o funcionamento dos sistemas agrícolas e alimentares em todo o mundo. A demanda reduzida por restaurantes e serviços de alimentação comercial, combinada com restrições de mão-de-obra, capacidade de processamento e/ou armazenamento de alimentos, levou os agricultores a desperdiçar grande quantidade da sua produção (Stephens et al., 2020). À medida em que a crise foi aumentando, esses impactos são sentidos de maneira mais profunda nos setores agrícolas e economias nacionais. A pandemia fez abrandar a economia mundial e, consequentemente, levou à redução de renda e à perda de empregos, afetando tanto o poder de compra quanto o acesso e disponibilidade de alimentos.

Desde o início da pandemia, nenhuma interrupção significativa no fornecimento de alimentos foi observada. No entanto, os desafios logísticos nas cadeias de suprimentos, particularmente as restrições de movimento domésticas e transfronteiriças, e as questões trabalhistas podem levar à interrupção no fornecimento de alimentos, especialmente se permanecerem a longo prazo (Ilo, 2020).

Além disso, restrições ao movimento podem impedir os agricultores de acessar mercados e resultar em desperdício de alimentos. Em muitos países, agricultores não conseguiram vender seus produtos nos mercados locais ou em escolas, restaurantes, bares, hotéis e outros estabelecimentos de lazer, uma vez que foram temporariamente fechados (Silva Filho \& Gomes Júnior, 2020). Assim, garantir o funcionamento contínuo das cadeias de suprimentos nacionais e globais é crucial para evitar uma crise alimentar nos países que já enfrentam desafios de SAN.

A maior procura e armazenamento de alimentos durante a pandemia, a redução ou interrupção da produção e distribuição de alimentos, além da escassez de mão-de-obra também podem resultar em picos de preços e aumento da volatilidade dos preços, desestabilizando os mercados internacionais. Mercadorias de alto valor, especialmente perecíveis como frutas e legumes frescos, carne, peixe e leite, são as mais afetadas (Galanakis, 2020).

No Brasil, os efeitos da pandemia nos preços dos alimentos foram revelados desde a segunda quinzena de março e a 
primeira de abril de 2020, mesmo período em que foi intensificado o isolamento social. Observou-se nesse intervalo o aumento dos preços dos alimentos in natura, ou minimamente processados, em relação à média dos alimentos em domicílios e outros grupos de alimentos da classificação NOVA. Os alimentos ultraprocessados, por outro lado, apresentaram uma inflação menor do que a média dos alimentos in natura (Brasil, 2020a).

Com a variação no preço dos alimentos, o perfil de compra e consumo também é modificado. Entre abril e maio de 2020, o Internacional Food Information Council realizou uma pesquisa com 1.011 consumidores americanos de 18 a 80 anos sobre o impacto da COVID-19 na compra de alimentos, comportamentos alimentares e percepções de segurança alimentar. A pesquisa mostrou que as pessoas fizeram menos compras pessoalmente e estavam comendo mais comidas caseiras durante o isolamento, enquanto $42 \%$ dos participantes da pesquisa relataram comprar mais alimentos embalados do que o habitual. Com relação à mudança dos hábitos alimentares, $43 \%$ dos participantes consideram seus hábitos alimentares mais saudáveis do que há alguns meses (18\% muito mais saudáveis, $25 \%$ um pouco mais saudáveis), enquanto outros $43 \%$ disseram que seus hábitos alimentares não mudaram (IFIC, 2020). Já no Brasil foi realizado um estudo transversal, com indivíduos maiores de 18 anos, também nos meses de abril e maio de 2020, que demonstrou uma diminuição da frequência de consumo de alimentos saudáveis, com maior redução no consumo regular de hortaliças, e um aumento na prevalência de consumo de alimentos não saudáveis (congelados, salgadinhos, doces e sobremesas) (Malta et al., 2020).

Apesar de não haver um desabastecimento generalizado de produtos, a redução da produção e os preços praticados reforça uma situação de insegurança alimentar, que não é resultado da falta de alimentos em si, mas da escolha dos alimentos no ato da compra. Pode-se estabelecer, portanto, uma tendência à constituição de hábitos alimentares inadequados, enquanto os alimentos ultraprocessados estão mais presentes na mesa da população, por meio da associação destes alimentos à ideia de conforto e prazer, como um alento aos efeitos da pandemia.

\section{Impacto no perfil nutricional}

Os impactos relacionados aos processos de disponibilidade (produção, comercialização e acesso ao alimento) durante a pandemia da COVID-19 têm provocado alguns desafios à escolha, preparo e consumo alimentar e sua relação com a saúde.

Alguns estudos buscaram conhecer as mudanças nos padrões de consumo alimentar da população antes e durante a pandemia da COVID-19. O estudo NutriNet Brasil encontrou um aumento no consumo de marcadores de alimentação saudável (vegetais, frutas e leguminosas) e estabilidade no consumo de marcadores de alimentos não-saudáveis (ultraprocessados) (Steele et al., 2020). Em um estudo realizado na Espanha, foi observado uma maior adesão à dieta mediterrânea durante o confinamento, entretanto, o consumo de alimentos não-saudáveis, como bebidas alcoólicas, lanches e doces, também aumentou (Sánchez-Sánchez et al., 2020). Uma pesquisa online realizada no Canadá com 254 famílias demonstrou que 60\% da amostra relatou alterações na alimentação e rotinas alimentares (mais tempo cozinhando e ingestão de mais salgadinhos, por exemplo) desde o início da pandemia (Carroll, 2020).

Sabe-se que o efeito das escolhas alimentares na saúde pode contribuir para a redução dos efeitos da COVID-19, pois alguns alimentos e nutrientes (cobre, folato, ferro, selênio, zinco e vitaminas A, B6, B12 e vitamina D) têm o potencial de melhorar a resposta imunológica (Gombart et al., 2020). No entanto, em um contexto de vulnerabilidade social, o acesso a estes nutrientes por grande parte da população está comprometido.

Um período de confinamento domiciliar e redução de atividade física desestrutura horários de refeições e de sono, aumentando o estresse emocional e o consumo de alimentos (Shen et al., 2020), o que pode aumentar o peso corporal. Um estudo realizado no Chile mostrou que hábitos alimentares saudáveis e atividade física regular podem ser fatores de proteção para o aumento de peso durante o confinamento devido à pandemia por COVID-19 (Reyes-Olavarría et al., 2020). Além disso, Ashby (2020) observou que, embora a alimentação não saudável tenha diminuído durante a pandemia, os declínios foram 
menos acentuados e a alimentação saudável parecia estar mais presente em populações com maior percentual de adultos com obesidade (Ashby, 2020). Assim, a otimização de cuidados e estratégias para o bem-estar geral das populações é fundamental durante esse período.

Em contrapartida, disgeusia e anosmia, sintomas descritos em indivíduos afetados com o novo coronavírus, estão intimamente relacionadas com os processos da perda de apetite, que podem promover a perda de peso, com consequente desnutrição (Giacomelli et al., 2020; Brann et al., 2020). Ressalta-se que pessoas desnutridas têm sistemas imunológicos mais debilitados e podem estar em maior risco de complicações graves decorrentes do Sars-CoV-2 (Pallath et al., 2021).

Certamente, a insegurança alimentar - seja grau leve, moderado ou grave - tem sido estabelecida com o surgimento da pandemia por COVID-19. A redução do poder de compra das famílias e a alta nos preços dos alimentos nos últimos tempos foram os principais fatores que levaram a reduções importantes no consumo alimentar, particularmente de alimentos nutricionalmente mais saudáveis (Cepal, 2018). Tudo isso, em meio a ambientes alimentares agressivos e repletos de alimentos ultraprocessados (fácil acesso e baixo custo), agrava a situação de inseguridade alimentar entre os mais vulneráveis (Monteiro et al., 2019).

A Rede PENSSAN divulgou, em abril de 2021, o inquérito nacional sobre insegurança alimentar no contexto da pandemia da COVID-19 no Brasil, com coleta de dados realizada em dezembro de 2020. O inquérito demonstrou que, nos três meses anteriores à coleta de dados, 44,8\% dos domicílios brasileiros tinham seus moradores em Segurança Alimentar. Dos demais, 55,2\% que se encontravam em Insegurança Alimentar; 9\% conviviam com a fome, ou seja, estavam em situação de insegurança alimentar grave, sendo pior essa condição nos domicílios de área rural (12\%). Portanto, do total de 211,7 milhões de brasileiros, 116,8 milhões conviviam com algum grau de Insegurança Alimentar e, destes, 43,4 milhões não tinham alimentos em quantidade suficiente e 19 milhões de brasileiros (as) enfrentavam a fome (Rede PENSSAN, 2021). Sendo assim, a combinação das crises econômica, política e sanitária provocou uma imensa redução da segurança alimentar em todo o Brasil, além do aprofundamento da situação de pobreza e de vulnerabilidade social vivenciada por muitas famílias brasileiras.

\section{Políticas dos países para acesso aos alimentos}

À medida em que a pandemia da COVID-19 forçava o fechamento de empresas em todo o mundo e coloca países em confinamento, os bloqueios adotados globalmente ameaçavam cortar cadeias de suprimentos e, assim, aumentar a insegurança alimentar. De acordo com a Food and Agriculture Organization (FAO), os governos podem proteger seus cidadãos mobilizando bancos de alimentos, oferecendo transferências monetárias a famílias vulneráveis, estabelecendo reservas alimentares de emergência e adotando medidas para proteger os trabalhadores agrícolas. Os países também devem expandir os programas de proteção social para ajudar aqueles que não tinham cobertura anteriormente (FAO, 2020a). Essas medidas de enfrentamento a pandemia auxiliam na garantia da segurança alimentar e nutricional da população mais vulnerável, além da garantia ao direito humano à alimentação adequada.

Nesse sentido, o Banco Mundial desenvolveu projetos e implantou financiamento de curto e longo prazo em alguns países da Ásia e África, a fim de garantir que as cadeias de suprimentos continuem abertas e funcionais. É concedido, então, apoio às populações mais vulneráveis por meio de programas de rede de segurança, complementados pela distribuição de alimentos em áreas onde as cadeias de suprimentos são severamente interrompidas (The World Bank, 2020).

Em Portugal, o Programa Operacional de Apoio às Pessoas Mais Carenciadas foi intensificado durante a pandemia para suprir a demanda de alimentos para famílias mais vulneráveis (POAPMC, 2020). Além disso, foi construída a Rede de Emergência Alimentar, estruturada a partir dos Bancos de Alimentos, para garantir o elo entre doadores e famílias em insegurança alimentar (Banco Alimentar, 2020). 
Outros países da Europa, como Alemanha, Espanha, França e Itália publicaram várias medidas de ajuda ao setor agrícola e alimentar, dentre elas o auxílio financeiro aos agricultores, às pequenas empresas e trabalhadores individuais; permissão da entrada de trabalhadores sazonais de outros continentes; e, no caso da Alemanha, autorização para que trabalhadores sazonais continuassem exercendo sua função sem contribuir para o sistema de seguridade social (USDA, 2020b; TMF Group, 2020). O governo da Alemanha, em 22 de março de 2020, também apresentou medidas para combater o impacto negativo na economia no país, incluindo apoio financeiro às famílias durante a crise. Além disso, os pais que perderam sua renda podem solicitar um suplemento de benefício infantil (Kinderzuschlag), que é pago além do abono familiar (Kindergeld) (Welt, 2020).

Nos EUA, ocorreu a criação do Coronavirus Food Assistance Program para fornecer assistência financeira quase imediata a produtores agrícolas. Além disso, o governo ampliou o Supplemental Nutrition Assistance Program, garantindo ajuda financeira às famílias e indivíduos de baixa renda para aquisição de alimentos por meio de vale-refeição. Ampliou também o acesso ao Special Supplement Nutrition Program for Women, Infants, and Children, que auxilia gestantes, lactantes e crianças menores de cinco anos nos cuidados de saúde e nutrição, fornecendo um cartão para compras de alimentos nutritivos. Os programas de Food Distribution também aumentaram seu campo de abrangência para que atendessem os indivíduos vulneráveis afetados pela COVID-19 (USDA, 2020a).

No Brasil, publicou-se a resolução de apoio aos agricultores familiares, além do governo ter tomado várias medidas de apoio a pequenas e médias empresas (Brasil, 2020b). Para trabalhadores informais e desempregados, o governo federal disponibilizou o auxílio emergencial para ajudar nas despesas básicas de uma família, além de oferecer a isenção de débitos de consumo de água e energia elétrica durante a pandemia por COVID-19. Em junho de 2020, o governo brasileiro promulgou a lei $n^{\circ}$ 14.016, que autoriza os estabelecimentos dedicados à produção e fornecimento de alimentos, produtos industrializados ou refeições prontas para o consumo, a doar os excedentes não comercializados e ainda próprios para o consumo humano sem maiores restrições (Brasil, 2020c). Entretanto, as iniciativas e políticas brasileiras para enfretamento da pandemia não consideram as diferentes dimensões da SAN, ou seja, não contém ações articuladas que contemplem tanto seu componente alimentar (disponibilidade, produção, comercialização e acesso aos alimentos) como nutricional (relacionado às práticas alimentares e utilização biológica dos alimentos).

Além disso, algumas políticas tomadas pelo governo atual contrapõem o componente alimentar da SAN, no âmbito do acesso aos alimentos, como liberação de fitossanitários (proibidos em outros países); o ataque constante ao atual guia alimentar da população brasileira; o fechamento das escolas públicas, o que dificultou o escoamento da produção e comercialização dos produtos do Programa de Aquisição de Alimentos; a ausência de punição no desmatamento da Amazônia, que culmina com o aumento das queimadas; além do favorecimento ao plantio de soja e arroz com finalidade de comercialização exterior e desabastecimento do mercado interno. Diante disso, as políticas atuais agravam a situação de fome e pobreza da população brasileira, repercutindo no aumento da insegurança alimentar e nutricional.

Em resumo, as políticas dos países que atendem às necessidades imediatas de segurança alimentar incluem: ampliar programas de redes de segurança, manter os alimentos em movimento, distribuir e melhorar o acesso aos alimentos, proteger empregos e meios de subsistência, apoiar a produção da próxima temporada, e os pequenos produtores locais.

\section{Experiências exitosas na pandemia em relação à Segurança Alimentar e Nutricional}

Em meio as consequências da pandemia para a SAN da população, é essencial que os países mantenham os sistemas alimentares funcionantes para que a crise da saúde não se transforme em crise alimentar.

A OMS, junto com seus parceiros, fornece orientações e aconselhamentos sobre SAN durante a pandemia aos governos, empresas de alimentos, profissionais de saúde e público em geral, objetivando manter uma boa saúde e prevenir a 
desnutrição em todas as suas formas (WHO, 2020). Várias universidades públicas também publicaram orientações e recomendações acerca da SAN durante o período da pandemia. O departamento de Nutrição da Universidade Federal do Rio Grande do Norte desenvolveu cartilhas, guias e e-books para o público em geral, abordando assuntos pertinentes à alimentação e nutrição no contexto da pandemia, como dicas e receitas para alimentação de crianças e adolescentes e orientações para serviços de delivery e alimentação escolar (UFRN, 2020). Apesar da importância desses materiais, eles não são acessíveis a população mais vulnerável, sendo divulgados apenas na internet e em mídias sociais.

Durante a pandemia, diversas organizações comunitárias, como escolas, conselhos escolares e voluntários em todo o mundo, desenvolveram programas inovadores para fornecer refeições, vouchers, cartões-presente ou caixas de alimentos frescos para estudantes e suas famílias, visto que programas alimentares para escolares não estavam disponíveis. No Brasil, ocorre a distribuição de alimentos aos pais ou responsáveis dos estudantes das escolas públicas por meio de kits alimentares adquiridos pelo Programa Nacional de Alimentação Escolar (Brasil, 2020d). Diferentemente do Brasil, no Canadá ainda não existe um Programa Nacional de Alimentação Escolar, contudo, ocorreu a distribuição de alimentos para as famílias de estudantes (Ryerson University, 2020).

Na cidade de Victoria, capital da Colúmbia Britânica no Canadá, os vereadores aprovaram uma proposta para expandir um programa de produção de alimentos urbanos, transferindo temporariamente alguns funcionários do departamento de parques para o cultivo de 50.000 a 75.000 mudas de plantas comestíveis para oferecer aos moradores. Desde março de 2020, as mudas crescem em viveiros e estufas municipais até que estejam prontas para a distribuição. Os residentes também recebem adubo, lascas de madeira e solo para cultivar seus alimentos em hortas domésticas, além de recursos educacionais, incluindo instruções para jardineiros iniciantes (Huffpost, 2020).

Outra experiência exitosa utilizada durante a pandemia é o kit de ferramentas do City Region Food System (CRFS), que foi desenvolvido no ano de 2018 pela RUAF Foundation (Resource Centres on Urban Agriculture and Food Security) e FAO, com o intuito de fornecer orientação sobre como avaliar e criar sistemas alimentares sustentáveis nas cidades. No contexto da pandemia, o kit de ferramentas pode ser aplicado para entender o impacto da COVID-19 nos sistemas alimentares e servir como base para planejamento e monitoramento (FAO, 2020b; RUAF, 2020a).

De um modo geral, as orientações podem ajudar a organizar os sistemas alimentares de maneira mais eficaz e sustentável a fim de atender às demandas de produtores e consumidores, promovendo a produção local de alimentos e cadeias de suprimentos. A longo prazo, a abordagem do CRFS ajudará a definir políticas, intervenções e mecanismos de governança territorial para a transformação do sistema alimentar, reforçando a cooperação e a colaboração entre os diferentes governos locais e outros atores, que é um dos elementos-chave para aumentar a resiliência do sistema alimentar local (RUAF, 2020b).

\section{Conclusão}

As medidas tomadas para reduzir a propagação do novo coronavírus impactou profundamente as condições de renda e emprego da população, afetando diretamente os sistemas alimentares mundiais. Mediante essa situação, é impreterível a necessidade de saídas que coloquem a vida e a dignidade humana no centro das decisões, a partir de políticas públicas que garantam os direitos humanos. As políticas públicas durante a pandemia também devem estar voltadas ao Direito Humano à Alimentação Adequada (DHAA), garantindo que todas as pessoas, especialmente as mais vulneráveis, tenham acesso a alimentos adequados e saudáveis.

Sendo assim, o desafio nesse momento é assegurar diferentes mecanismos que contribuam para garantir o DHAA, potencializando diferentes estratégias de abastecimento alimentar. Além disso, são necessárias iniciativas de educação alimentar e nutricional que orientem e estimulem a adoção/manutenção de hábitos alimentares saudáveis a toda família, sempre considerando as medidas de precaução sanitária. 


\section{Referências}

Ashby, N. J. S. (2020). The impact of the COVID-19 pandemic on unhealthy eating in populations with obesity. Obesity (Silver Spring), $28,1802-1805$.

Banco Alimentar (2020). Rede de emergência alimentar: ajudar não pode parar. Portal Eletrônico do Banco alimentar. https://www.bancoalimentar.pt/quemsomos/pagina-noticias/noticias-federacao/rede-de-emergencia-alimentar-ajudar-nao-pode-parar/

Bartley, M. (2017). Health inequality: an introduction to concepts, theories and methods. (2 ${ }^{\mathrm{a}}$. ed.) Cambridge, UK, Malden, MA, USA: Polity Press.

Bastos, L. S. (2020). COVID-19 e hospitalizações por SRAG no Brasil: uma comparação até a 12a semana epidemiológica de 2020 . Cad. Saúde Pública, 36 (4).

Brann, D. H., Tsukahara, T., Weinreb, C., Logan, D. W., \& Datta, S. R. (2020). Non-neural expression of SARS-CoV-2 entry genes in the olfactory epithelium suggests mechanisms underlying anosmia in COVID-19 patients. Sci. Adv., 6.

Brasil (2020a). Instituto Brasileiro de Geografia e Estatística (IBGE). Índice Nacional de Preços ao Consumidor Amplo 15 - IPCA-15. https://sidra.ibge.gov.br/home/ipca15/brasil.

Brasil (2020b). Lei no 14.048, de 24 de agosto de 2020. https://www.in.gov.br/en/web/dou/-/lei-n-14.048-de-24-de-agosto-de-2020-273920579

Brasil (2020c). Lei n ${ }^{\circ}$ 14.016, de 23 de junho de 2020. http://www.in.gov.br/web/dou/-/lei-n-14.016-de-23-de-junho-de-2020-263187111

Brasil (2020d). Lei no 13.987, de 07 de abril de 2020. https://www.in.gov.br/en/web/dou/-/lei-n-13.987-de-7-de-abril-de-2020-251562793

Carroll, N., Sadowski, A., Laila, A., Hruska, V., Nixon, M., Ma, D. W. L., \& Haines, J., On Behalf Of The Guelph Family Health Study (2020). The Impact of COVID-19 on Health Behavior, Stress, Financial and Food Security among Middle to High Income Canadian Families with Young Children. Nutrients, 12 (8).

Comissão Econômica para a América Latina e o Caribe (CEPAL) (2018). Panorama Social de América Latina. https://www.cepal.org/ptbr/publicaciones/44412-panorama-social-america-latina-2018-documento-informativo.

Comissão Econômica para a América Latina e o Caribe (CEPAL) (2020a). Coyuntura Laboral en América Latina y el Caribe. El trabajo en tiempos de pandemia: desafíos frente a la enfermedad por coronavirus (COVID-19). Relatório 22.

Comissão Econômica para a América Latina e o Caribe (CEPAL) (2020b). Panorama social de América Latina. https://www.cepal.org/pt$\mathrm{br} /$ comunicados/pandemia-provoca-aumento-niveis-pobreza-sem-precedentes-ultimas-decadas-tem-forte

Food and Agriculture Organization of the United Nations (FAO) (2020a). The State of Food Security and Nutrition in the World 2020. Transforming food systems for affordable healthy diets. Rome, FAO.

Food and Agriculture Organization (FAO) (2020b). Urban Food Actions Platform. http://www.fao.org/urban-food-actions/knowledge-products/resourcesdetail/en/c/1133524/

Galanakis, C. M. (2020). The Food Systems in the Era of the Coronavirus (COVID-19) Pandemic Crisis. Foods, 9 (4), 523.

Giacomelli, A., Pezzati, L., Conti, F., et al. (2020). Self-reported olfactory and taste disorders in SARS-CoV-2 patients: a cross-sectional study. Clin Infect Dis, $71(15), 889-890$.

Gombart, A. F., Pierre, A., \& Maggini, S. (2020). A Review of Micronutrients and the Immune System-Working in Harmony to Reduce the Risk of Infection. Nutrients, 12 (1).

House, J., \& Williams, D. R. (2000). Understanding and reducing socioeconomic and racial/ethnic disparities in health. In: Smedley, B.D. \& Syme, S.L. (org.). Promoting health: intervention strategies from social and behavioral research. Washington: National Academy Press, pp. 89-131.

Huffpost (2020). Victoria Assigns Parks Staff To Start Growing Food For Residents. https://www.huffingtonpost.ca/entry/victoria-seedlingscoronavirus_ca_5e8819e6c5b6cbaf28296c57?fbclid=IwAR005oUMIWwAthYLfoMh6j_oP6_bLyPGjkejVQrrFDYeHaWxJ4E_-nML8b4\&guccounter=2

International Food Information Council (IFIC) (2020). Food \& Health Survey. www.foodinsight.org.

International Labour Organization (ILO) (2020). COVID-19 and the world of work: Impact and policy responses. Monitor 1st Edition.

Malta, D. C., Szwarcwald, C. L., Barros, M. B. A., et al. (2020). A pandemia da COVID-19 e as mudanças no estilo de vida dos brasileiros adultos: um estudo transversal, 2020. Epidemiol. Serv. Saúde, 29(4).

Monteiro, C. A., Cannon, G., Levy, R. B., Moubarac, J. C., Louzada, M. L. C., Rauber, F., et al. (2019). Ultra-processed foods: What they are and how to identify them. Public Health Nutr, 22 (5), 936-941.

Pallath, M. M., Ahirwar, A. K., Tripathi, S. C., Asia, P., Sakarde, A., \& Gopal, N. (2021). COVID-19 and nutritional deficiency: a review of existing knowledge. Horm Mol Biol Clin Invest., 42 (1), 77-85.

Programa Operacional de Apoio às Pessoas Mais Carenciadas (POAPMC) (2020). Portal Eletrônico do POAPMC. https://poapmc.portugal2020.pt/inicio

Quinzani, M. A. D. (2020). O avanço da pobreza e da desigualdade social como efeitos da crise da covid-19 e o estado de bem-estar social. Boletim de Conjuntura (BOCA) ano II, 2 (6).

Rede brasileira de pesquisa em soberania e segurança alimentar e nutricional (Rede PENSSAN) (2021). VIGISAN - Inquérito nacional sobre insegurança alimentar no contexto da pandemia da Covid-19 no Brasil. 66p. 
Reyes-Olavarría, D., Latorre-Román, P. Á., Guzmán-Guzmán, I. P., Jerez-Mayorga, D., Caamaño-Navarrete, F., \& Delgado-Floody, P. (2020). Positive and Negative Changes in Food Habits, Physical Activity Patterns, and Weight Status during COVID-19 Confinement: Associated Factors in the Chilean Population. Int J Environ Res Public Health, 17 (15), 5431.

Rocher, E. T. (2007). Revisão sistemática x revisão narrativa. Acta Paulista de Enfermagem, 20 (2).

Ryerson University (2020). Examining emergent school food models during and after COVID-19. Portal Eletrônico da Ryerson University. https://www.ryerson.ca/foodsecurity/projects/activity_examining_emergent_school_food_models_covid19/

RUAF (2020a). City Region Food Systems to cope with COVID-19 and other pandemic emergencies. https://ruaf.org/news/city-region-food-systems-to-copewith-covid-19-and-other-pandemic-emergencies/

RUAF (2020b). City Region Food System Toolkit. https://ruaf.org/news/city-region-food-system-toolkit/

Sánchez-Sánchez, E., Ramírez-Vargas, G., Avellaneda-López, Y., Orellana-Pecino, J. I., García-Marín, E., \& Díaz-Jimenez, J. (2020). Eating Habits and Physical Activity of the Spanish Population during the COVID-19 Pandemic Period. Nutrients, 12 (9).

Santos, J. A. F. (2020). Covid-19, causas fundamentais, classe social e território. Trabalho, Educação e Saúde, 18 (3).

Shen, W., Long, L. M., Shih, C. H., \& Ludy, M. J. (2020). A Humanities-Based Explanation for the Effects of Emotional Eating and Perceived Stress on Food Choice Motives during the COVID-19 Pandemic. Nutrients, 12 (9).

Silva Filho, O. J., \& Gomes Júnior, N. N. (2020). O amanhã vai à mesa: abastecimento alimentar e COVID-19. Cad. Saúde Pública, 36 (5).

Stephens, E. C., Martin, G., van Wijk, M., Timsina, J., \& Snowe, V. (2020). Editorial: Impacts of COVID-19 on agricultural and food systems worldwide and on progress to the sustainable development goals. Agricultural Systems, 183.

Steele, E. M., Rauber, F., Costa, C. D. S., Leite, M. A., Gabe, K. T., Louzada, M. L. D. C., Levy, R. B., \& Monteiro, C. A. (2020). Dietary changes in the NutriNet Brasil cohort during the covid-19 pandemic. Rev Saude Publica, 54 (91).

The World Bank (2020). Food Security and COVID-19. Portal Eletrônico do The world bank. https://www.worldbank.org/en/topic/agriculture/brief/foodsecurity-and-covid-19

TMF Group (2020). Government support schemes for COVID-19. Portal Eletrônico do TMF Group. https://www.tmf-group.com/en/newsinsights/coronavirus/government-support-schemes/

United States Department of agriculture (USDA) (2020a). Food Assistance. Portal Eletrônico do USDA. https://www.usda.gov/coronavirus/foodassistance\#food-distribution

United States Department of agriculture (USDA) (2020b). German COVID-19 Related Aid Measures for the Farm and Food Sector. Report.

Universidade Federal do Rio Grande do Norte (UFRN) (2020). https://sigaa.ufrn.br/sigaa/public/departamento/documentos.jsf?lc=pt_BR\&id=203.

Welt (2020). Eltern stoßen bei notwendigen Einkäufen auf Aggression. Portal Eletrônico do Welt. https://www.welt.de/politik/deutschland/article206751915/Corona-Was-Familien-leisten-muessenist-ein-gigantisches-Sozialexperiment.htm

World Health Organization (WHO) (2020). Nutrition and Food Safety (NFS) and COVID-19. https://www.who.int/teams/nutrition-and-food-safety/covid-19

World Health Organization (WHO) (2021a). Critical preparedness, readiness and response actions for COVID-19: Interim guidance. https://www.who.int/publications/i/item/critical-preparedness-readiness-and-response-actions-for-covid-19.

World Health Organization (WHO) (2021b). WHO Coronavirus (COVID-19) Dashboard. https://covid19.who.int/. 